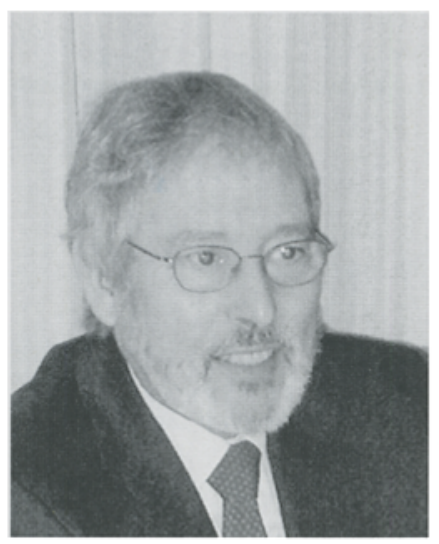

Rolf Harbeck

\section{Von Kontinuität und Verpflichtung}

Liebe Leserin, lieber Leser,

mit dem neuen Jahr 2004 und Heft 1 der Kartographischen Nachrichten beginnt - selbstverständlich - ein never Jahrgang unserer Zeitschrift. Er ist der Vierundfünfzigste und damit Ausdruck einer Kontinuität, die nicht unbedingt zu den Selbstverständlichkeiten unserer kurzlebigen Zeit gehört. Wohl ist in den zurückliegenden Jahren manche neve Fachzeitschrift entstanden, doch manche ist auch bald wieder verschwunden und andere haben sich zusammengeschlossen oder ihr Profil total umgestaltet. Dass eine Fachzeitschrift Rückschau halten kann auf über fünfzig laufende Erscheinungsjahrgänge, darf als Erfolg und als Zeichen von Willenskraft verstanden werden. Ohne jeden Zweifel aber ist es Verpflichtung.

Verpflichtet fühlen sich Vorstand und Vorstandsrat der Deutschen Gesellschaft für Kartographie und natürlich die Schriftleitung der KN. Gemeinsam gilt es, eine Fachzeitschrift für Geoinformation und Visualisierung - auch wenn dies nur ihr Untertitel ist - weiter zu etablieren und auszubauen. Dazu gehören äußere Zeichen, wie diese, dass die DGfK sich zu ihrer Verantwortung als Herausgeberin auch in Titel und Impressum bekennt und dass gleichzeitig die enge Verbundenheit der deutschsprachigen kartographischen community in gemeinsamer Organschaft von Osterreich, der Schweiz und Deutschland zum Ausdruck kommt. Dazu gehört aber vor allem, dass unser Fachblatt in Inhalt und Auftritt die zukunftsweisenden Entwicklungslinien der Zeit weiterhin aufgreift und umsetzt.
Dies können und wollen wir nicht abrupt tun, sondern behutsam und schrittweise. Oberster Anspruch bleibt, dass wissenschaftlich bedeutende und innovative, begutachtete Aufsätze sich mit journalistisch geprägten Fachbeiträgen aus der Praxis abwechseln. Dies kann im normalen gemischten Heft geschehen, dazu gibt es aber auch die Möglichkeit der Themenhefte. Dass wir hierbei in begrenzter Zahl auch englischsprachige Originalbeiträge veröffentlichen wollen, ist eine Verpflichtung gegenüber der jungen Generation. Sie hat einen Anspruch darauf, Internationalität in ihrer Zeitschrift ebenso vorzufinden wie die Möglichkeit zur Auseinandersetzung mit englischer Fachidiomatik und -terminologie. Und dass wir unermüdlich daran arbeiten werden, die KN ganz in Farbe herauszubringen, sind wir ihrem zum Anspruch erhobenen Untertitel schuldig: Denn zur Visualisierung gehört das farbige (kartographischel Bild, nicht das in Schwarz-Weiß verfremdete.

Innerhalb unserer fachlichen und kollegialen Gemeinschaft ist der Dialog wichtig. Hierzu hat der Vorstand der DGFK eine eigene Kolumne erhalten, in der er regelmäßig aus seiner Arbeit berichten wird. Sie wird auch unseren Schweizer und Österreichischen Kollegen offen stehen. Der Schriffleiter kann Sie, liebe Leserinnen und Leser, im Editorial ansprechen. Zum Dialog aber brauchen wir vor allem Sie, die Sie die KN in Händen halten und sich zu Debatte und Kritik, zu Veränderung oder Bestätigung herausgefordert fühlen. Deshalb: Bitte schreiben Sie uns!

Herzlich, Ihr

\section{RoY GAOCuch}

